\title{
THE YERKES OBSERVATORY PHOTOELECTRIC
}

\author{
PARALLAX SCANNER
}

\author{
W. F. VAN ALTENA \\ Yerkes Observatory, Williams Bay, Wis., U.S.A.
}

\begin{abstract}
A photoelectric parallax scanner designed and constructed at the Yerkes Observatory to measure parallaxes and proper motions at the telescope is described. The concept of the parallax scanner is similar to Griffin's radial velocity spectrometer except that the parallax star and the field stars are monitored separately, thus making the technique purely differential. Observations with the 40-in. refractor under less than ideal conditions gave the error of a single measurement as $\pm 4.5 \mu$ (s.e.). Observations under clear skies and with improved drive and guidance should decrease the error by a factor of 2 to 3 .
\end{abstract}

A prototype instrument designed to measure parallaxes and proper motions photoelectrically at the telescope has been constructed at the Yerkes Observatory. The concept of the parallax scanner is similar to Griffin's (1967) radial velocity spectrometer except that the problem is two dimensional. In Griffin's spectrometer a photographic positive of a stellar spectrogram is placed in the focal plane and scanned to and fro to yield the position of maximum transmission, which can be related to the stellar radial velocity relative to standard stars observed on the same night. The Yerkes Observatory photoelectric parallax scanner, which has been briefly described earlier (van Altena, 1972), utilizes a high contrast contact positive of a recent photographic plate of the stellar field in question. The mask is completely opaque except for the star 'holes', thus minimizing the night sky light due to the Moon, etc.

In Figure 1, which is an optical schematic of the parallax scanner, light from the 40 -in. refractor objective is focused on the mask; when the mask is in registration with the stellar field, light is transmitted by the mask and imaged by the $140 \mathrm{~mm}$ lucite field lens on two photomultipliers, which are located in a cold box. The central $12 \mathrm{~mm}$ of the lucite lens has been cored out and beveled on the flat side to deviate the light from the central (parallax) star to an off axis photomultiplier, while light from the field stars is imaged on the central photomultiplier. The signals from the two photomultipliers are amplified and then recorded on a two-pen chart recorder. Figure 2 shows the tailpiece of the 40 -in. refractor with the parallax scanner mounted on the automatic guiding camera.

The lower scan shown in Figure 3 is of $\mathrm{BD}+42^{\circ} 3123$, a star of magnitude $V=8.34$, while the upper scan is of the field. In order to retain a small image diameter on the mask, $\mathrm{BD}+42^{\circ} 3123$ was reduced to $V=12.3$ by a 4 -mag. sector on the mask plate. The scans shown in Figure 3 were made on a night with light cirrus, the effects of which can be seen in the tracings. Much of the noise in the tracings is due to telescope drive errors and 'hunting' in the automatic guider, about $\pm 4 \mu \mathrm{rms}$, which is unimportant in the photographic work. We are making a number of improvements in the telescope drive and guider to minimize the hunting since it is deleterious to the parallax scanner observations. The scan rate used at the present time is a slow 


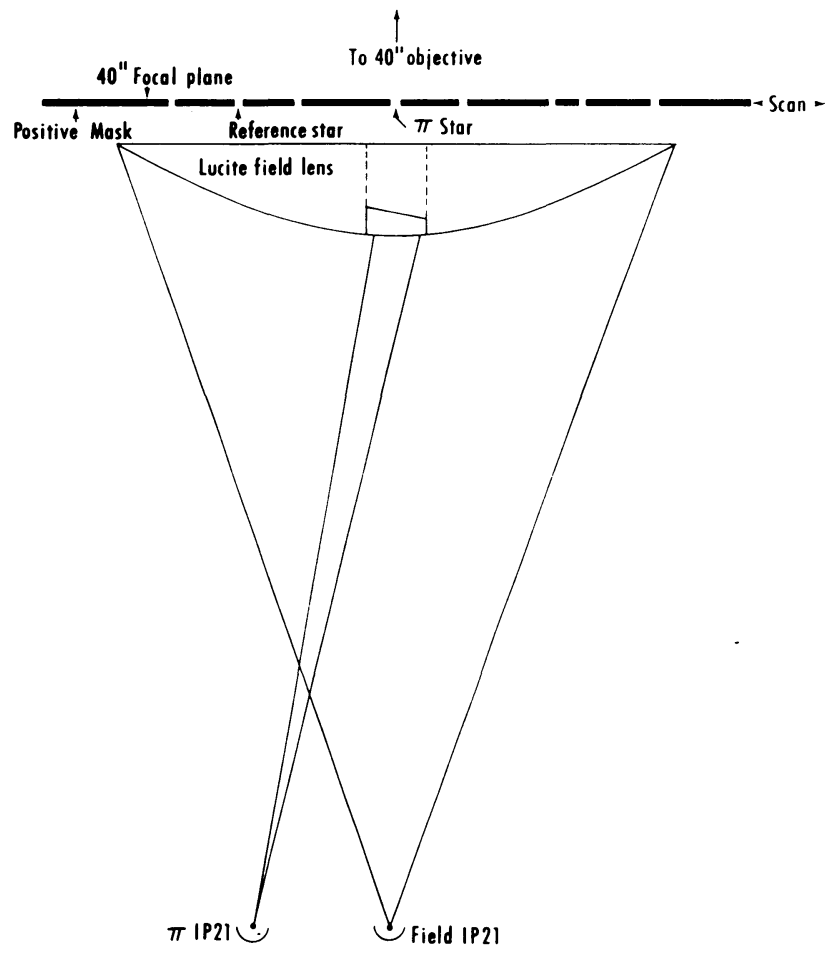

Fig. 1. Optical schematic of the Yerkes Observatory parallax scanner. See text for explanation.

$1^{\prime \prime} \min ^{-1}$ of time in order to average out guiding errors and fluctuations in seeing.

Measurement of the position of the central star relative to the field is done by measuring the peak displacements on the chart paper and multiplying by the appropriate scale factor, which is $5.2 \mu /$ chart $\mathrm{mm}$ in this case. Based on the interagreement from six scans of this star an error of $\sigma_{1}= \pm 4.5 \mu$ (s.e.) for the relative position from a single scan was found. This is about two times poorer than we can do photographically at the present time at Yerkes, where $\sigma_{1}= \pm 2.3 \mu$ (s.e.) is average. However, once the guiding errors have been minimized and given clear weather it should be possible to reduce the parallax scanner error to less than $2 \mu$ for a single scan. Further improvements could undoubtedly be made through pulse counting and computer analysis.

Aside from the high potential accuracy of the technique, due to its purely differential nature, a very large gain has been achieved in reducing the effort involved in parallax determinations. A parallax series with the parallax scanner would typically consist of perhaps six scans on a night with perhaps 25-30 nights spread over 2-3 years as is presently the case for most Yerkes parallaxes. However, the tracings can be measured and reduced in a few minutes in contrast to the large amount of time 


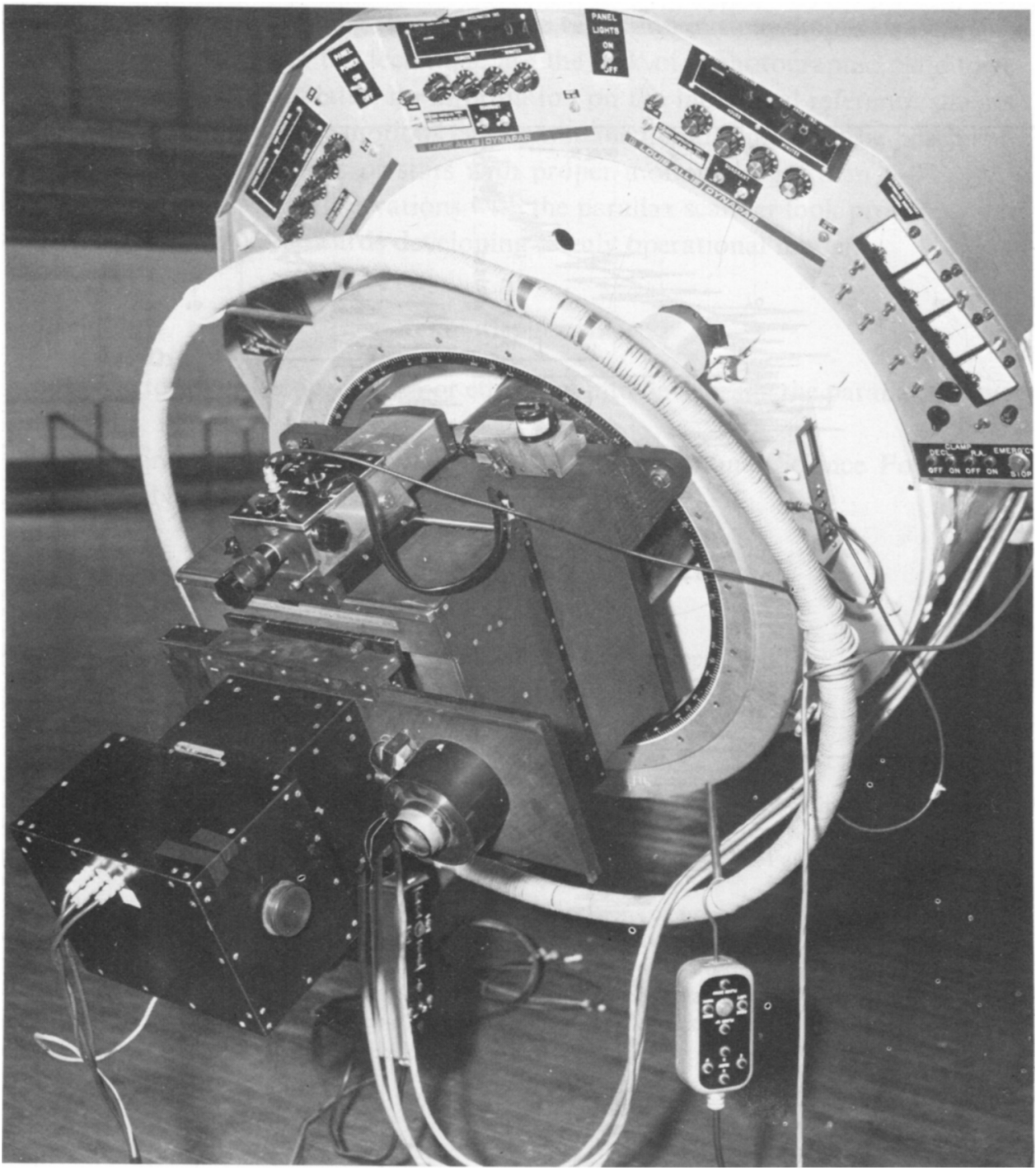

Fig. 2. The tailpiece of the 40-in. refractor showing the parallax scanner mounted on the automatic guiding camera. 


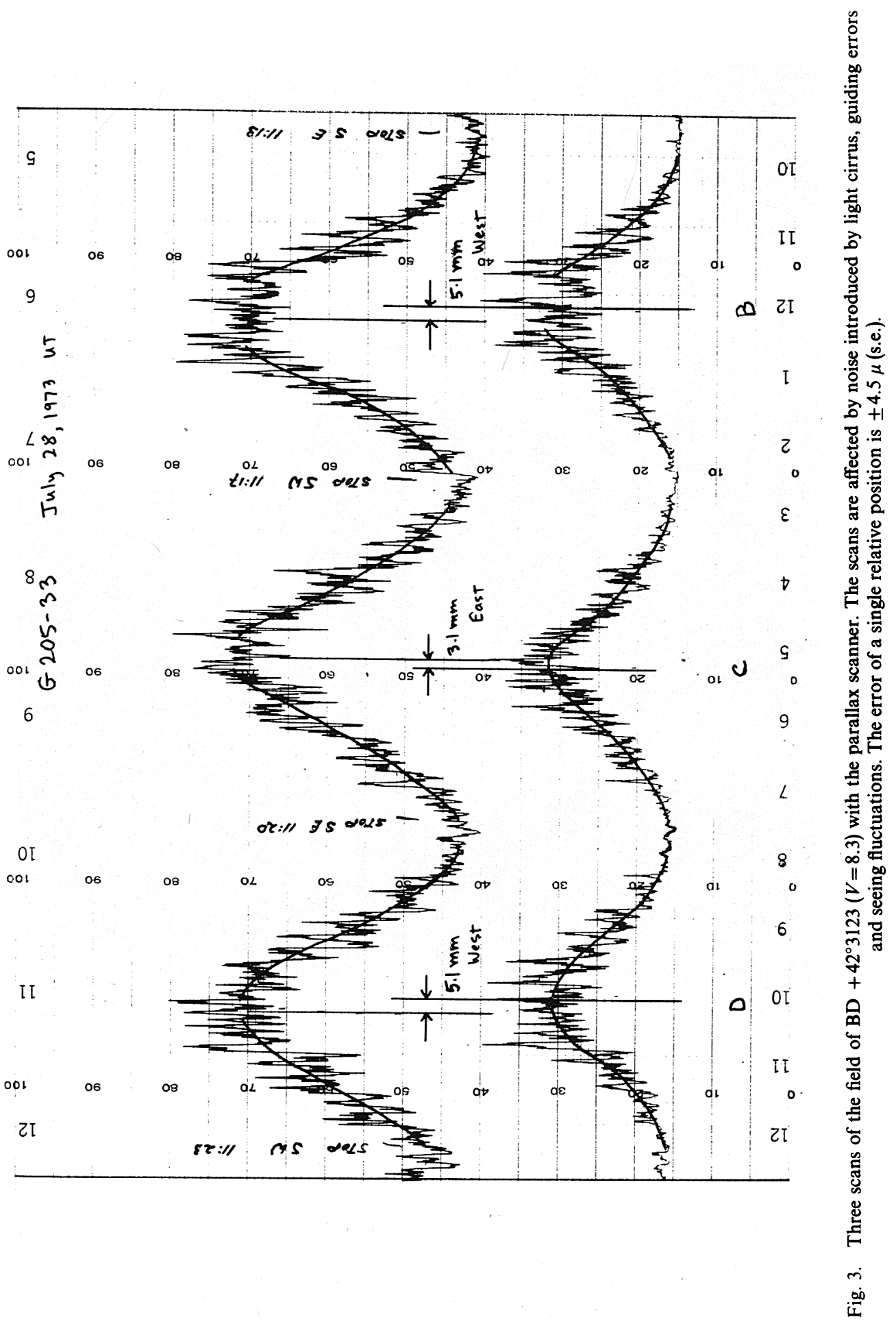


spent measuring and reducing the plates; the reduction in time should be $\sim 90 \%$.

Some disadvantages of the technique are the lack of a photographic plate to reanalyze perhaps 50 years later, no information on the individual reference star relative parallaxes and proper motions can be obtained, and it may not be possible to observe either close binaries or stars with proper motions larger than $\sim 0$ ". $2 \mathrm{yr}^{-1}$. Despite these limitations observations with the parallax scanner look promising and work will be continued towards developing a truly operational model.

\section{Acknowledgements}

I would like to acknowledge the major effort put into developing the parallax scanner by Robert J. Pernic.

This research has been supported in part by the National Science Foundation under grant NSF GP-13771.

\section{References}

Griffin, R. F.: 1967, Astrophys. J. 148, 465.

Van Altena, W. F.: 1972, Bull. Am. Astron. Soc. 4, 11. (Observatory reports.)

\section{DISCUSSION}

Moffet: Griffin's method for measuring radial velocities is useful because the same mask can be used for hundreds of stars, for example in a cluster. I fail to see the advantage of your system, which requires a special mask for each field. Is it in any way better than conventional plates and an automatic measuring engine?

Van Altena: Since it is necessary to make about 25 observations of a parallax star over a period of 2-3 years the requirement for a new mask for each field is not too burdensome. Advantages of the parallax scanner are that the observations are purely differential and not semi-absolute as in the radial velocity spectrometer, and that even with automatic measuring machines the amount of labor involved in the measuring and reductions to determine a high quality parallax is excessive. The parallax scanner can reduce this effort by about $90 \%$. 